\title{
Brief report - Improving Hand Hygiene in a resource limited setting - a Malawian Example
}

\section{A.Thumbs ${ }^{1}$, M.Lingomanje ${ }^{2}$ and C.Rothe ${ }^{3}$}

1 Department of Surgery, Queen Elizabeth Central Hospital, Blantyre, Malawi.

2 Infection Control Practitioner, Queen Elizabeth Central Hospital, Blantyre, Malawi.

3 Department of Medicine, Queen Elizabeth Central Hospital, Blantyre, Malawi

\section{The Problem:}

Hand hygiene is the key tool to prevent healthcare-associated infections and hence to protect the health of patients, guardians and health care workers. In the developed world infection prevention programs that include hand-hygiene as a basic means have been implemented in every single hospital decades ago but this is not yet true for many countries in the developing world where infection control policies are not in place or are poorly funded and limited resources pose a major challenge. In developed countries $5-10 \%$ of hospital in-patients are estimated to acquire a nosocomial infection. Little is known yet about the importance of nosocomial infections in developing countries, but estimates put the risk at $3-12 \%$. Hand hygiene is the simplest and perhaps least expensive way to prevent healthcare-associated infections. The WHO highlighted the importance of hand hygiene by launching a campaign in 2009 and by issuing guidelines for resource-restrained settings.

\section{Introducing alcohol based hand rub at Queen Elizabeth Central Hospital - a possible solution:}

Alcohol-based hand rub is a possible step to improve the hygiene situation in such a setting and was recommended already in 2002 by CDC. This paper gives an example of the first started project in Malawi.

Queen Elizabeth Central Hospital is constantly overrun by large amounts of patients. The wards are overcrowded, floor beds a common sight, maintenance of sinks and water supply is a constant problem. During wards rounds doctors examine many patients whereby proper hand washing after each patient is impossible. Most of patient care is done by guardians with sometimes very limited knowledge about hand hygiene. In a charity-funded project 50 wall dispensers were purchased and installed on ten selected medical and surgical wards and on the Intensive Care Unit (ICU) of QECH. After intense discussion with pharmacy, hospital administration and the Central Medical Stores of Malawi a sustainable supply of methylated spirit and glycerin could be secured. Spirit and glycerin were mixed in the hospital pharmacy (11 spirit plus 10mg glycerin) and stored in 51 containers. Together with the Infection Control Practitioner at QECH two focal persons per ward were identified and trained in a workshop. The participants were taught basic facts about hand hygiene and about the importance of spreading the knowledge to new members of staff, medical students and - probably most essentially - to guardians who carry the major burden of patient care in Malawi. The wall dispensers were demonstrated and the participants were taught about handling and care of the dispensers. The idea was to render these focal persons responsible for the refill of the dispensers. Furthermore measures of hygiene and infection prevention, stressing the fact that hygiene should not end at the hospital gates but instead be continued after discharge. Information material in Chichewa and English was produced to help everyone on the wards in question understand how and when to use alcoholic hand rub. Typical scenes like wound care, emptying of urine bags, feeding etc. were depicted on photographs. At least twice per week the focal persons were meant to train staff, students and guardians about the use of alcoholic hand rub and about general possibilities improving hand hygiene at hospital and at home.

This is the first time in Malawi that alcoholic hand rub has been made available on a larger scale, involving not only health care workers and students, but also guardians. This campaign will be framed by a research project on nosocomial and surgical site infections where base line data prior to the introduction of hand rub will be compared with results after one and two years of use of hand rub.

\section{Introducing alcohol based hand rub at Queen Elizabeth Central Hospital - is it a success?}

Improving hand hygiene is possible even under difficult circumstances. A major task is to include not only health care workers but also guardians who play a crucial role in patient care. To achieve and maintain the sensitive use of hand rub and other hygiene measures intensive training will be needed in future. A major challenge during this project was that trained focal persons often were moved away from the wards without our knowledge, so that new staff had to be trained all over again. Also the intended teaching of new staff in the ward and guardians is not taking place as often as wished and necessary. Furthermore supply at Pharmacy could not be maintained at all times due to shortage of personal. It should be discussed if each hospital should have at least one hygiene focal person whose main job it could be to mix the hand rub, refill and clean the dispensers and to teach staff and guardians. This seems a much easier way forward.

Universal access to alcoholic hand rub in wall dispensers should be standard of care in all hospitals in Malawi. Spirit and glycerin should become an integral part of every hospital budget. Training of staff and guardians has to go on continuously.

The project outlined above will provide information on how to establish improvements in hand hygiene and may help to convince stakeholders at ministerial level and contribute to a more large scale roll-out of hand hygiene practices in Malawi and other resource-limited settings.

\section{References}

1. Ponce-de-Leon-Rosales S. and Macias A., Global perspectives of invection control. In: R.P. Wenzel, Editor, Prevention and control of nosocomial infections (4th ed.), Lippincott Williams\&Winks, Philadelphia (2003), pp. 14-33

2. Lynch P., Pittet D., Borg M.A. and Methar S., Infection control in countries with limited resources. J. Hosp. Infect 65 (2007), pp. 148150

3. Nyamogoba H, Obala AA. Nosocomial infections in developing countries: cost effective control and prevention. East Afr Med J 2002;79(8):435-41

4. Pittet D., Boyce J.M., Hand hygiene and patient care: pursuing the Semmelweis legacy. Lancet Infect Dis 2001; 4: 9-20 